\title{
Niobium in the spectra of metal-poor stars
}

\section{Laimons Začs}

Faculty of Physics and Mathematics, University of Latvia, Raina bulv. 19, Riga, LV-1586, Latvia E-mail: zacsdatnet.LV

\section{Arturs Barzdis*}

Faculty of Physics and Mathematics, University of Latvia, Raina bulv. 19, Riga, LV-1586, Latvia E-mail: arturs 1vdinbox.1v

\section{Martiņš Sandars}

Faculty of Physics and Mathematics, University of Latvia, Raing bulv. 19, Riga, LV-1586, Latvia E-mail: martins.sandarsdgmail.com

\section{Elvijs Matrozis}

Faculty of Physics and Mathematics, University of Latvia, Rainga bulv. 19, Riga, LV-1586, Latvia E-mail: e-mateinbox.1v

\begin{abstract}
Abundances of neutron-capture elements are calculated in the atmospheres of three metal-poor stars and the standard star Arcturus with a special emphasis to niobium. The method of spectral synthesis, carefully compiled line lists and recent data of hyperfine splitting for $\mathrm{Nb}$ I lines are used. The results and possible origin of niobium in the analyzed metal-poor stars are discussed.
\end{abstract}

11th Symposium on Nuclei in the Cosmos, NIC XI

July 19-23, 2010

Heidelberg, Germany

\footnotetext{
* Speaker.
} 


\section{INTRODUCTION}

The chemical elements heavier than iron are created by a combination of slow (s) and rapid (r) neutron-capture nucleosynthesis processes [1]. The theory of nucleosynthesis identifies different astrophysical sites for s- and r-processes. The r-process nuclei are the products formed primarily during the evolution of massive stars and supernova explosions. The s-process nuclei are generally thought to have been synthesized during late stages of stellar evolution of low-mass stars. Recents studies that argue two separate r-processes are responsible for the production of the heavier and lighter neutron capture elements ([2], and references therein). The detection of lighter neutron capture elements ( $38 \leq \mathrm{Z} \leq 48$ ) in the spectra of metal-poor stars is crucial for determining whether two different r-processes indeed exist. To date the niobium abundance was calculated only in the atmospheres of a few stars using a couple of identified lines in high-resolution absorption spectra $[3,4,5,6]$. Future studies of abundance patterns of neutron-capture elements including niobium are encouraged to create a statistically significant sample of metal poor stars. The recent measurements of transition probabilities and hyperfine splitting reported for some elements (see, for example, $[7,8])$ should be incorporated.

A reliable abundance of niobium in the solar photosphere was determined in [9] using original radiative lifetimes of $\mathrm{Nb}$ II lines. Equivalent widths of eleven $\mathrm{Nb}$ II lines were measured in the solar spectrum to calculate the niobium abundance, $\log \varepsilon(\mathrm{Nb})=\log \left(N_{N b} / N_{H}\right)+12.00=1.42 \pm 0.06$. The hyperfine structure splitting was neglected in these estimates. The latest review of the standard solar composition (SAD) recommends the value $\log \varepsilon(\mathrm{Nb})=1.42 \pm 0.06$, which is in good agreement with the meteoritic abundance, $\log \varepsilon(\mathrm{Nb})=1.39 \pm 0.03$ [10]. Kwiatkowski et al. [11] obtained a much higher solar niobium Abundance, $\log \varepsilon(\mathrm{Nb})=2.10 \pm 0.10$, using $\mathrm{Nb}$ I lines. The most plausible explanation of such a discrepancy seems to be the uncertainty in measurements of equivalent widths for weak and broad $\mathrm{Nb}$ I lines disturbed by hyperfine splitting. Thus, the hyperfine structure can have a significant effect on stellar absorption line profiles and the corresponding abundances can be substantially overestimated if such effects are not taken into account in the calculations [12].

\section{OBSERVATIONS AND ANALYSIS}

High resolution spectra of three metal-poor stars (see Table 1) were obtained on August 24th 2008 with the optical echelle spectrograph FIES installed at the $2.5 \mathrm{~m}$ Nordic Optical Telescope (NOT) on La Palma with a resolving power of $R=67000$ and $S / N>100$. The spectra cover a wavelength range from about 3700 to $7300 \AA$. The Visible and Near Infrared Atlas of the Arcturus spectrum [13] was used for the comparison Star Arcturus. For the data reduction, the Pythonand PyRAF-based data reduction software package FIEStool was used. The LTE analysis program ABUNDANCE available together with the spectral synthesis program SPECTRUM written by Corbally \& Gray [14] was used to calculate abundances. Atmospheric models were taken from the ATLAS9 model atmosphere grid [15]. For spectral synthesis the SPECTRUM and STARSP code and atomic line data from VALD [16] and DREAM [17] databases were used.

The ratio of light to heavy neutron capture elements was calculated: $[\mathrm{hs} / \mathrm{ls}]=[\mathrm{hs} / \mathrm{Fe}]-[\mathrm{ls} / \mathrm{Fe}]$; $[\mathrm{ls} / \mathrm{Fe}]=1 / 4([\mathrm{Sr} / \mathrm{Fe}]+[\mathrm{Y} / \mathrm{Fe}]+[\mathrm{Zr} / \mathrm{Fe}]+[\mathrm{Nb} / \mathrm{Fe}]) ;[\mathrm{hs} / \mathrm{Fe}]=1 / 5([\mathrm{Ba} / \mathrm{Fe}]+[\mathrm{La} / \mathrm{Fe}]+[\mathrm{Ce} / \mathrm{Fe}]+$ 
Table 1: Basic data for observed stars and the comparison star Arcturus.

\begin{tabular}{lcccccccr}
\hline Star & Sp.type & $M_{V}$ & $\begin{array}{c}T_{\text {eff }} \\
(\mathrm{K})\end{array}$ & $\begin{array}{c}\log g \\
(\mathrm{cgs})\end{array}$ & $\begin{array}{c}\xi_{t} \\
\left(\mathrm{~km} \mathrm{~s}^{-1}\right)\end{array}$ & {$[\mathrm{Fe} / \mathrm{H}]$} & {$[\mathrm{hs} / \mathrm{ls}]$} & $\log \varepsilon(\mathrm{Nb})$ \\
\hline HD209621 & C1,2 CH & $-1.9 M_{\text {bol }}$ & 4500 & 1.5 & 1.9 & -1.8 & +0.5 & \\
HD218732 & G6/G8 Ib & -2.8 & 4200 & 0.5 & 2.4 & -1.5 & -0.1 & 0.30 \\
HD232078 & K3 IIp & -2.2 & 4000 & 0.5 & 2.0 & -1.5 & -0.2 & 0.35 \\
Arcturus & K1.5 III & -0.3 & 4300 & 1.5 & 1.4 & -0.5 & & 0.74 \\
\hline
\end{tabular}

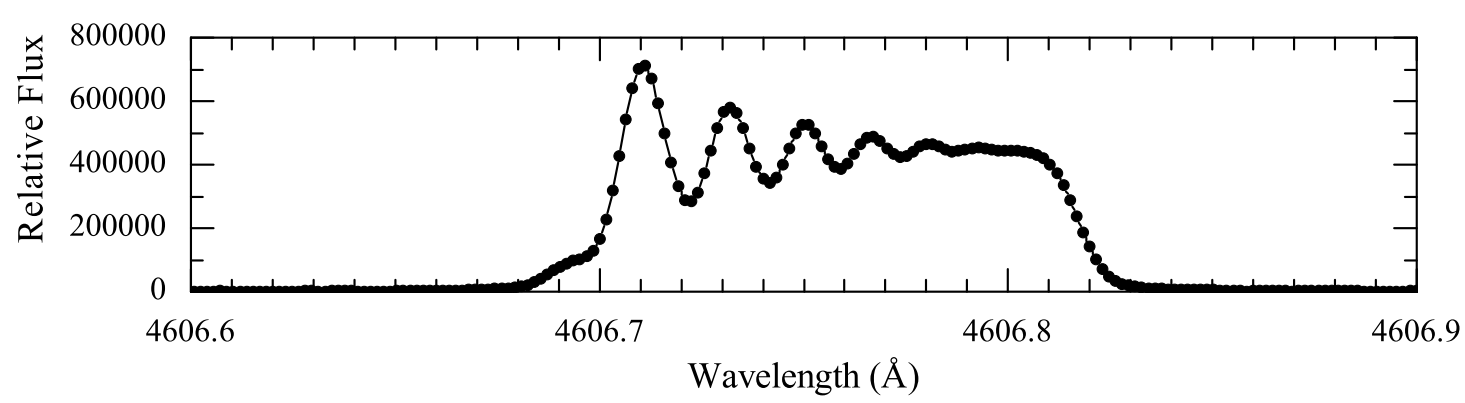

Figure 1: Fourier transform spectrum of $\mathrm{Nb}$ I line at $4606.756 \AA$ according [8], showing its hyperfine structure.

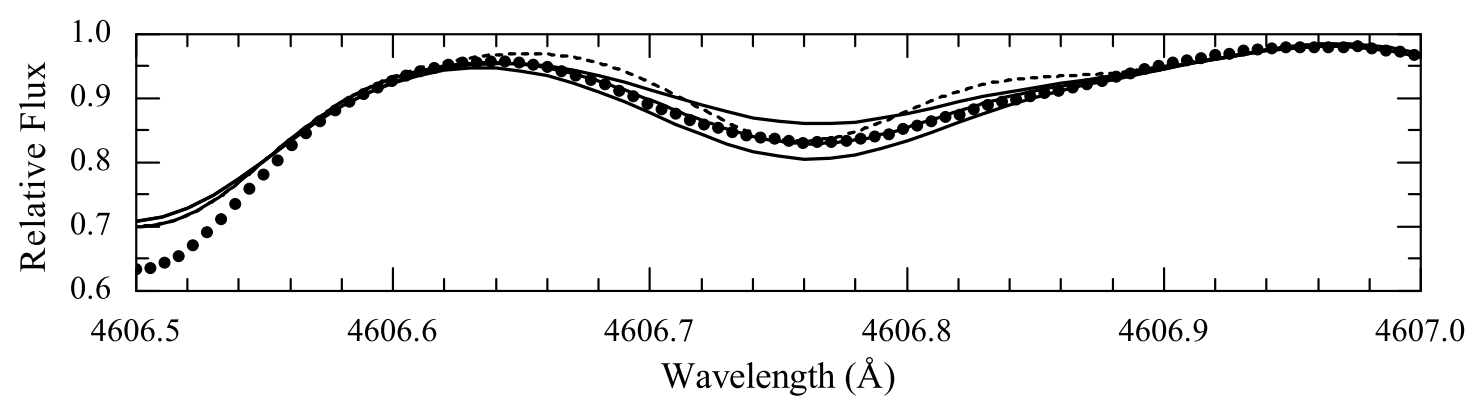

Figure 2: Observed spectrum (filled circles) of Arcturus around the Nb I line at $4606.756 \AA$ along with the synthesized profile without (dashed line) and with HFS correction (solid lines) calculated for the final niobium abundance: $\log \varepsilon(\mathrm{Nb})=0.65$ and $0.74 \pm 0.1 \mathrm{dex}$.

$[\mathrm{Nd} / \mathrm{Fe}]+[\mathrm{Sm} / \mathrm{Fe}])$

\section{RESULTS AND CONCLUSIONS}

Abundance of niobium in the atmosphere of Arcturus was calculated using four relatively unblended $\mathrm{Nb} \mathrm{I}$ lines including HFS correction (see Figure 1 and 2). Abundance ratio $[\mathrm{Nb} / \mathrm{Fe}]=-0.06$ is close to the solar and meteoritic abundance [10].

Abundance of niobium is slightly enhanced in the atmospheres of HD218732 and HD232078 relative to the Sun, $[\mathrm{Nb} / \mathrm{Fe}] \approx+0.4$ (see Figure 3 ). The abundance ratio of the heavy s-process peak elements to the light s-process peak elements, [hs/ls] was found to be in the range -0.1 to -0.2 dex (see Table 1). The distribution of neutron-capture elements are well described by the solar r-process abundance curve, suggesting a large r-process contribution. 


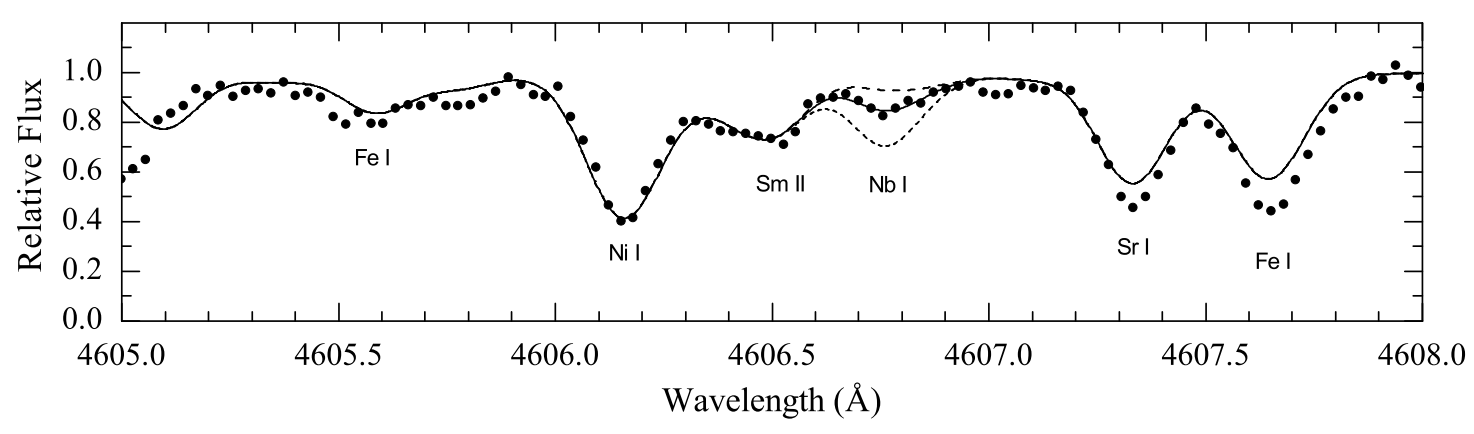

Figure 3: The observed spectrum (filled circles) of HD232078 around Nb I line at $4606.756 \AA$ along with synthesized spectra for three different niobium abundances: $\log \varepsilon(\mathrm{Nb})=+0.35 \pm 0.5$.

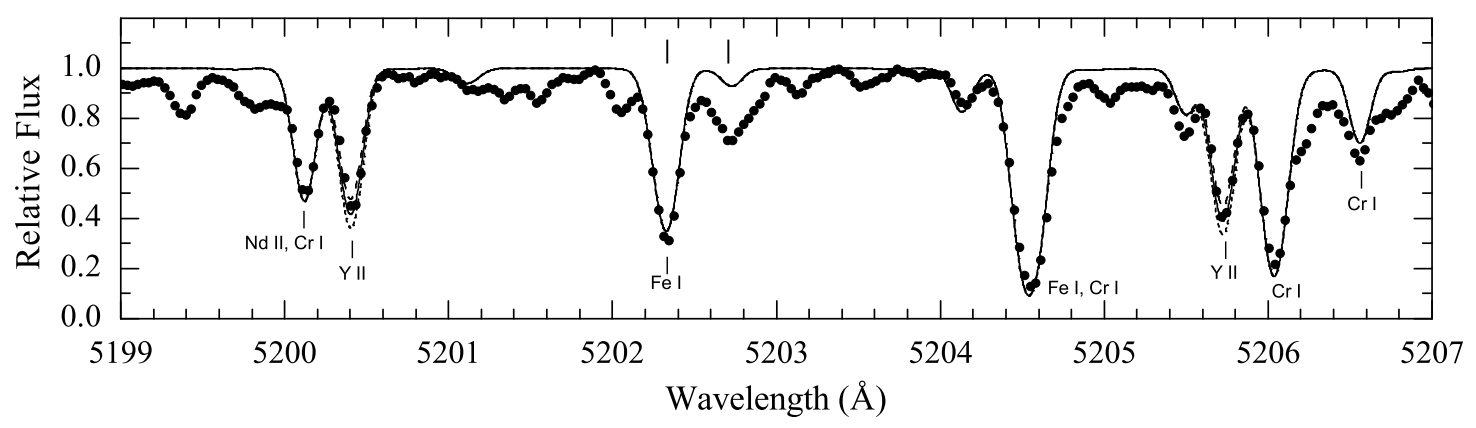

Figure 4: Observed spectrum (filled circles) of HD209621 along with synthesized profiles of important atomic lines calculated using the final atmospheric parameters and abundances for three $\mathrm{Y}$ abundances: $\log \varepsilon(Y)=1.26 \pm 0.3 \mathrm{dex}$. The positions of CN Red system lines are marked by vertical ticks.

The mean abundances for 28 elements are calculated in the atmosphere of HD209621 and neutron-capture elements are found to be significantly enhanced. Calculations of abundances for $\mathrm{Y}, \mathrm{Ce}$, and $\mathrm{Nd}$ are illustrated in Figures 4 and 5. Unfortunately, the synthesis of weak niobium lines was plagued because of blending with strong lines of carbon bearing molecules $\left(\mathrm{CN}, \mathrm{C}_{2}\right.$, etc.) and therefore it was not possible to determine a reliable $\mathrm{Nb}$ abundance. The abundance ratio of the heavy s-process peak elements to the light s-process peak elements was found to be higher for HD209621, [hs/ls] $=+0.5$, close to the mean value found for CH-stars. Long-period radial velocity variations are confirmed for HD209621 by McClure [18]. Thus, enhanced neutroncapture elements in the atmosphere of HD209621 seems to be the result of mass transfer in the past from the companion star of higher initial mass (which is now a white dwarf).

\section{References}

[1] E.M. Burbidge, G.R.Burbidge, W.A.Fowler, F.Hoyle, Synthesis of the elements in stars, 1957, RvMP, 29, 547.

[2] C.Sneden, J.J.Cowan, R.Gallino, Neutron-capture elements in the early Galaxy, 2008, ARA\&A, 46, 241.

[3] A.V.Yushchenko, V.F.Gopka, C.Kim, Y.C., Liang, F.A.Musaev, G.A.Galazutdinov, The chemical composition of the mild barium star HD202109, 2004, A\&A, 413, 1105. 


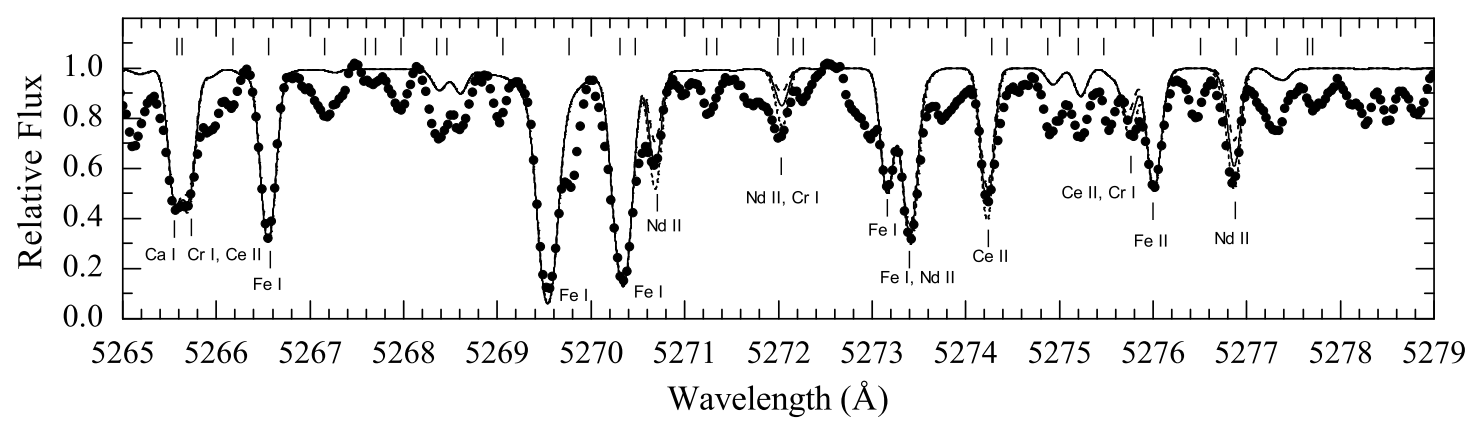

Figure 5: Observed spectrum (filled circles) of HD209621 along with synthesized profiles of important atomic lines calculated using the final atmospheric parameters and abundances for three different $\mathrm{Ce}$ and $\mathrm{Nd}$ abundances: $\log \varepsilon(\mathrm{Ce})=1.48 \pm 0.3 \mathrm{dex}$ and $\log \varepsilon(\mathrm{Nd})=1.18 \pm 0.3 \mathrm{dex}$. Positions of CN Red system lines are marked by vertical ticks.

[4] I.Ivans, C.Sneden, R.Gallino, J.J.Cowan, G.W.Preston, Near-ultraviolet observations of CS29497-030: new constraints on neutron-capture nucleosynthesis process, 2005, ApJ, 627, L145.

[5] S.Honda, W.Aoki, Y.Ishimaru, Y.Wanajo, S.G.Ryan, Neutron-capture elements in the very metal poor star HD122563, 2006, ApJ, 643, 1180.

[6] A.Barzdis, High resolution spectroscopy of two metal-poor red giants: HD232078 and HD218732, 2010, MNRAS, 408, 1452.

[7] H.Nilsson H., S.Ivarsson, Experimental oscillator strengths and hyperfine constants of NbII, 2008, $A \& A, 492,609$.

[8] S.Kroger, A.Er, I.K.Ozturk, G.Basar, A.Jarmola, R.Ferber, M.Tamanis, L.Začs, Hyperfine structure measurements of neutral niobium with Fourier transform spectroscopy, 2010, A\&A, 516, A70.

[9] P.Hannaford, R.M.Lowe, E.Biemont, N.Grevesse, Radiative lifetimes for Nb II and the problem of the solar abundance of niobium, 1985, A\&A, 143, 447.

[10] N.Grevesse, M.Asplund, A.J.Sauval, The solar chemical composition, 2007, Space Sci. Rev., $130,105$.

[11] Kwiatkowski M., Zimmermann P., Biemont P., Grevesse N, New lifetimes for NbI and RhI and the solar photospheric abundances of $\mathrm{Nb}$ and $\mathrm{Rh}, 1982, A \& A, \mathbf{1 1 2}, 337$.

[12] A.J. Booth, D.E. Blackwell, The effect of hyperfine structure on stellar abundance analysis,1983, MNRAS, 204, 777

[13] K.Hinkle, L.Wallace, J.Valenti, D.Harmer, Visible and near infrared atlas of the Arcturus spectrum 3727 - 9300 A, 2002, San Francisco:ASP

[14] C.J.Corbally, R.O.Gray, The late-B stars: Refined MK classification with stromgren photometry and the effects of rotation, 1994, AJ, 107, 1556

[15] F.Castelli, R.L.Kurucz, New grids of ATLAS9 model atmospheres, in proceedings of the 210th Symposium of the IAU held at Uppsala Modelling of stellar atmospheres, 2003

[16] F. Kupka, T.A. Ryabchikova, N.E. Piskunov, H.C. Stempels, W.W. Weiss, VALD-2 - The New Vienna Atomic Line Database, 2000, Baltic Astronomy, 9, 590

[17] E. Biémont, P. Palmeri, P. Quinet, A New Database of Astrophysical Interest, 1999, Ap\&SS, 269, 635

[18] R.D.McClure, The binary nature of the CH stars, 1984, ApJ, 280, L31. 